\title{
Improvement of Reconstruction Resolution of Computer-generated Hologram Using Phase Freedom
}

\author{
Daiki Yasukochi ${ }^{\text {a }}$ Yuki Misaki ${ }^{a}$, Yosuke Koga ${ }^{a}$, Shiyuan Yang ${ }^{a^{*}}$ \\ ${ }^{a}$ Kyusyu Institute of Technology, 1-1 Sensui-cho Tobata-ku Kitakyusyu-shi Fukuoka-ken, 804-8550, Japan \\ *Corresponding Author: yang@elcs.kyutech.ac.jp
}

\begin{abstract}
In this paper, we describe the simulation results of improving the resolution of the reconstructed image using the phase freedom in the original image in the Fourier transform amplitude hologram. Since the thing that is recorded in the amplitude hologram is the amplitude information, the phase of the original image is possible to arbitrarily specify. Utilizing the phase freedom can reconstruct an image more clearly by reducing the error of reconstructed image. Therefore, we can expected to improve the resolution of the reconstructed image.
\end{abstract}

Keywords: hologram, random phase, speckle.

\section{Introduction}

A hologram is a product that is applied to the holographic techniques that is a technique for reconstructing the original three-dimensional image from the information recorded by the object beam from the three-dimensional object. The light has an amplitude and phase information. But a hologram cannot be recorded only one. The thing that is recorded only the amplitude information is amplitude hologram. The original three-dimensional images are reconstructed based on this amplitude information. The computer-generated hologram is a method of calculating the amplitude distribution of the light diffracted and transmitted from the object and reconstructs the image on the computer. By utilizing the computer-generated hologram, the recording of the amplitude information becomes easy. There is also an advantage of being able to create a hologram for the fictitious three-dimensional objects.

Generally, in order to reconstruct the image clearly, it is necessary to use a hologram that is sufficiently large number of pixels. However, the number of hologram pixels is limited basically, so there is a limit of the image that can be reconstructed clearly. Therefore, in the amplitude hologram of the same number of pixels we try to reconstruct the clearer image by utilizing the phase freedom of the original image and improving resolution of the reconstruction image.

As we use the phase freedom of the original image, the initial phase information of the inputted three-dimensional object is random. This phase information is called the random phase. On the other hand, when we reconstruct the hologram of a three-dimensional object that has the random phase as the initial phase information, dark spots noise called speckle appears on the reconstruction image. We get a clearer reconstruction image by introducing iterative speckle reduction method that is combined speckle reduction method and iterative dummy area method ${ }^{(2)(3)}$ for optimizing the hologram so that the error of the reconstruction image is reduced with removing the speckle.

\section{The Fourier transform amplitude hologram in computer-generated hologram}

\subsection{Amplitude hologram}

Light has the amplitude and phase information. Hologram is possible to reconstruct the original threedimensional image by recording them. However, since it is difficult to record the amplitude and phase information at the same time, a hologram is usually recorded only either. The hologram that is recorded amplitude information is the amplitude hologram. The amplitude hologram reconstructs the original three-dimensional image by only the amplitude information. Therefore, the reconstruction image includes some errors because the phase information is lost.

\subsection{Fourier transform hologram}

Fourier transform hologram is a hologram that was 


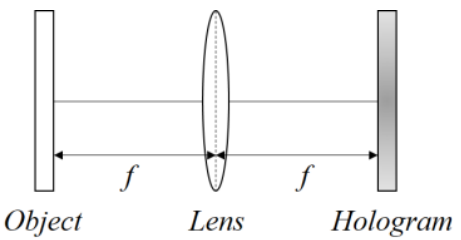

(a) Recording

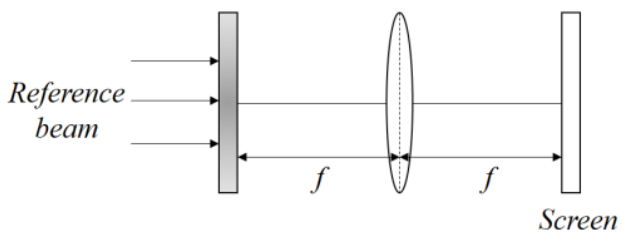

(b) Reconstruction

Fig. 1. Optical system of the Fourier transform hologram.

created by utilizing a Fourier transform action of the lens We can get a Fourier transform action of the lens by placing the object that we want to record at the position of the front focus point of lens, whose focus length is defined as $f$. We can get the Fourier transform hologram by recording the amplitude information of the object beam. And we can get a reconstruction image of the Fourier transform hologram by exposing a hologram to a plane wave and utilizing the Fourier transform action to the diffracted light again. Figure 1 shows the optical system of the Fourier transform hologram.

\subsection{Computer-generated hologram}

Fourier transform computer-generated hologram is created by calculating the object beam using the Fourier transform on a computer and obtaining the amplitude distribution at the hologram. There is an advantage of being possible to calculate the object beam at a high speed because of being able to use a Fast Fourier Transform on a computer.

\section{Iterative speckle reduction method}

\subsection{Iterative dummy area method}

As described in section 2.1, some errors are occurred in the reconstruction image because the amplitude hologram has no phase information. In iterative dummy area method, we set the area whose initial value of the amplitude and phase around the input original image is zero. This area is called dummy area. We will reduce the errors of the reconstruction image by adjusting the amplitude and phase of the dummy area so that the errors of the reconstruction image is reduced. The technique for searching optimal solution of the amplitude and phase of the dummy area in iterative, such as

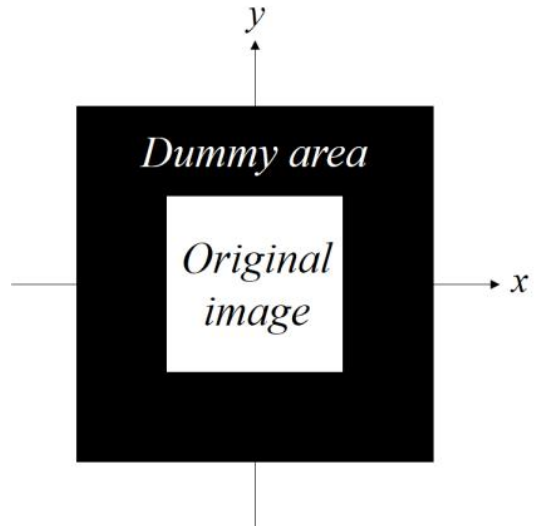

Fig. 2. Dummy area.

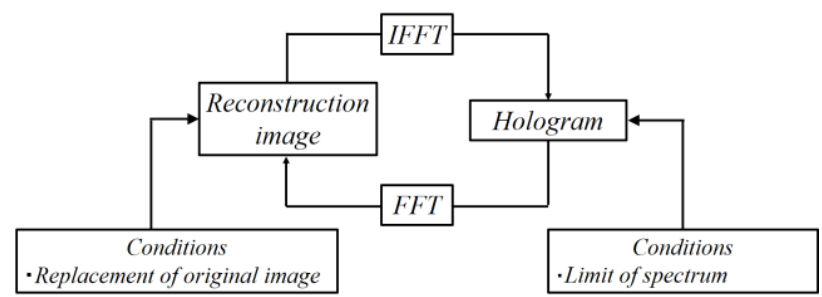

Fig. 3. Flow of repeat dummy area method.

the errors of the reconstruction image is reduced, is iterative dummy area method. Figure 3 shows the flow of iterative dummy area method.

\subsection{Speckle reduction method}

(a) Cause of speckle

Cause of speckles is related to the phase difference between the adjacent discrete 4 points of the reconstruction image, as shown in Fig 4. The phase $\varphi$ on the hologram is $-\pi \leq \varphi<\pi$ and each of the phase $\varphi$ is defined as $\varphi(x, y), \varphi(x-1, y), \varphi(x, y-1)$ and $\varphi(x-1, y-1)$ on the basis of the lower right. We also are defined as follows the phase difference of adjacent 4 points.

$$
\begin{aligned}
& \Delta \varphi_{1}=\varphi(x, y)-\varphi(x, y-1) \\
& \Delta \varphi_{2}=\varphi(x-1, y)-\varphi(x, y) \\
& \Delta \varphi_{3}=\varphi(x-1, y-1)-\varphi(x-1, y) \\
& \Delta \varphi_{4}=\varphi(x, y-1)-\varphi(x-1, y-1)
\end{aligned}
$$

The sum of these 4 phase difference $\Delta \varphi_{i}(i=1,2,3,4)$ is obviously zero. Also, since each of the phase difference become range of $-2 \pi \leq \Delta \varphi_{i}<2 \pi$, that is two cycles, It is necessary to limit the range of $-\pi \leq \varphi<\pi$ by operating the phase. This is called wrapping. The sum of the phase difference after wrapping becomes $2 \pi,-2 \pi$ and $-4 \pi$ in addition to zero. When the sum of the phase difference after wrapping is $2 \pi,-2 \pi$ and $-4 \pi$, the speckles occur.

(b) Cause of speckle

When the sum of the phase difference after wrapping 


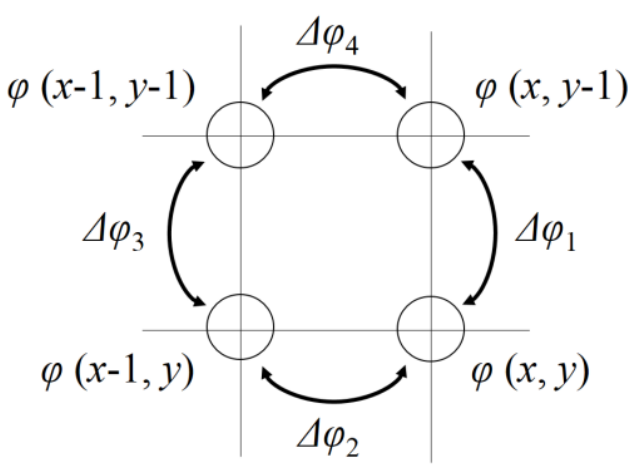

Fig. 4. Cause of speckle.

becomes $2 \pi,-2 \pi$ and $-4 \pi$ as section $3.2(a)$, we operate the phase for only one point in the adjacent 4 points so that the sum of the phase difference after wrapping becomes zero. It is possible to remove the speckles by using this speckle reduction method in the iterative dummy area method. This is called iterative speckle reduction method.

\section{Simulation and results}

We simulated the two original images under the following conditions.

- Original images : $64 \times 64$ pixels

- Dummy area : 4 times $(256 \times 256$ pixels $)$

- Band limit : $1 / 2$

- Hologram : $256 \times 256$ pixels

- Number of iteration : 2000times

The result of figure 6 is not used the iterative speckle reduction method and the initial phase is zero, that is not used the phase freedom of the original image. The result of figure 7 is used the iterative speckle method of 2000 times iteration and the initial phase is the random phase, that is used the phase freedom of the original image. When we compare figure 6 and 7, in both $\mathrm{F}$ and Lenna reconstruction image, it can be seen that the result of figure 7 is clearer than one of figure 6. Especially, in the Lenna image, the difference is obvious. We think that Lenna image had many highfrequency components than the F image, so the place of improvement were many by the phase freedom of the original image. From this results, it is shown that the improvement of the resolution of the reconstruction image is possible by taking the initial phase for the random phase and utilizing the phase freedom of the original image with using iterative speckle reduction method. In addition, the thing like streaks that appear in the reconstruction image is called Gibbs phenomenon. This occurs in a point that has a particularly high frequency components. We think that this

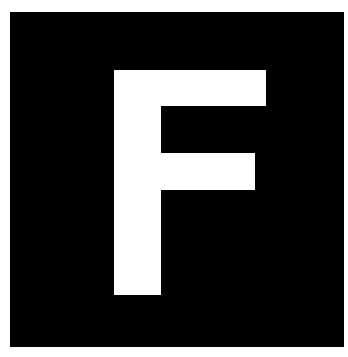

(a) $\lceil\mathrm{F}\rfloor$

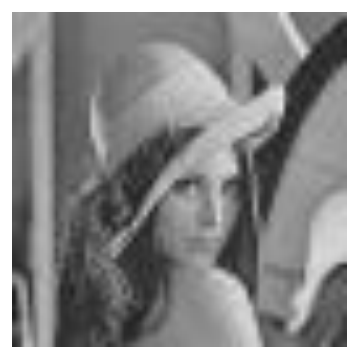

(b) $\lceil$ Lenna $\rfloor$
Fig. 5. Original image.

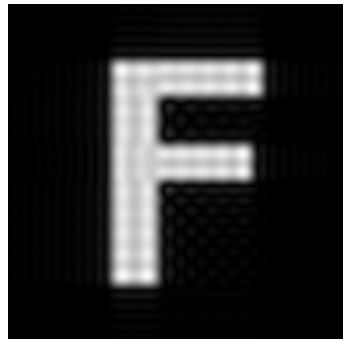

(a) $\lceil\mathrm{F}\lrcorner$

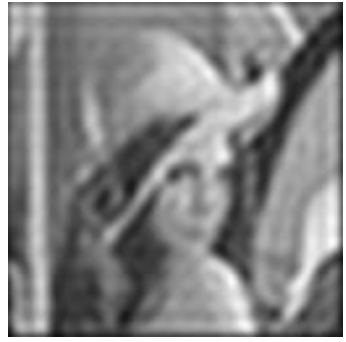

(b) $\lceil$ Lenna $\rfloor$
Fig. 6. Reconstruction image. (non-phase freedom)

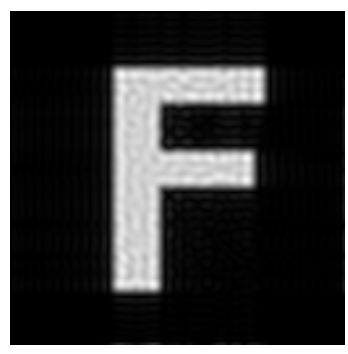

(a) $\lceil\mathrm{F}\lrcorner$

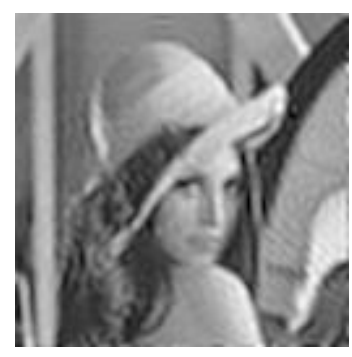

(b) $\lceil$ Lenna $\rfloor$
Fig. 7. Reconstruction image. (phase freedom)

is improved by increasing the number of iteration.

\section{Conclusion}

Generally, in order to reconstruct the image clearly, it is necessary to use a hologram that is sufficiently large number of pixels. However, the number of hologram pixels is limited basically, so there is a limit of the image that can be reconstructed clearly. Therefore, we simulated the clear reconstruction image by using the phase freedom of the original image and improving the resolution of the reconstruction image. The initial phase of the original image was the random phase in order to use phase freedom of the original image. And the random phase occurs speckle, so we introduced iterative speckle reduction method.

In the simulation, it was confirmed that the reconstruction image that is adapted to iterative speckle 
reduction method using the random phase in the initial phase becomes clearer. Thus, it was shown that the approach that utilizes a phase freedom of the original image using the iterative speckle reduction method and taking the initial phase for the random phase can improve the resolution of the reconstruction image.

\section{References}

(1) P. Hariharan : "Basics of Holography", pp. 4-12, 2004

(2) Toshinori Hora and Shiyuan Yang : "Iterative Dummy Area Method with Flexible Dummy Area Size for the Design of Kinoform" Journal of the Institute of Industrial Applications Engineers, Vol. 1, No. 2, 69-72, 2013

(3) Shiyuan Yang and Hiroaki Takajo "Speckle Reduction of Kinoform Reconstruction Utilizing the $2 \pi$ Ambiguity of Image Phase Differences" Optical Review, Vol. 12, No. 2, 93-96, 2005 